CASO CLÍNICO

\title{
Criptorquidectomía laparoscópica en dos perros utilizando bisturí ultrasónico
}

\author{
Laparoscopic cryptorchidectomy in two dogs using ultrasonic \\ scalpel
}

\begin{abstract}
Carlos Hernández L, ${ }^{1 *}$ M.Sc.
${ }^{1}$ Universidad CES, Facultad de Medicina Veterinaria y Zootecnia. Grupo de Investigación INCA-CES. Medellín, Colombia. *Correspondencia: chernandez@ces.edu.co
\end{abstract}

Recibido: Enero de 2012; Aceptado: Diciembre de 2012.

\section{RESUMEN}

Tradicionalmente, los perros con testículos retenidos abdominales son remitidos para orquiectomía a través de laparotomía media ventral o paraprepucial. Sin embargo, las técnicas mínimamente invasivas realizadas a través de equipos endoscópicos, son una alternativa excelente para efectuar el procedimiento, debido a las ventajas en la reducción del trauma tisular y un menor dolor posoperatorio, además de la reducción de complicaciones posquirúrgicas entre otras ventajas. A continuación se presentan dos casos de pacientes caninos criptórquidos intervenidos quirúrgicamente mediante laparoscopia, para la extracción de los testículos retenidos en las que además se utilizó un bisturí ultrasónico. En Colombia, el desarrollo de las técnicas endoscópicas en cirugía de pequeños animales aún se encuentra muy limitado, y probablemente esta sea la primera publicación en el país acerca del uso de laparoscopia en casos de orquiectomía en pacientes con testículos retenidos en la cavidad abdominal.

Palabras clave: Criptorquidismo, laparoscopia, perro (Fuente: CAB).

\begin{abstract}
Traditionally, dogs with abdominally retained testicles are submitted to orchiectomy throughout a ventral midline or parapreputial laparotomy. However, minimally invasive techniques performed using endoscopic equipment are an excellent alternative for the abdominal procedure, because of the advantages in tissular trauma reduction, less postoperative pain and reduction in complications among other advantages. Two cases of abdominal cryptorchidectomy performed using minimally invasive techniques and ultrasonic scalpel in dogs, are presented in this report. In Colombia, the development of minimally invasive techniques in small animal surgery is still very limited, and this is possibly the first publication in the country regarding the use of this technique in two cases of abdominal cryptorchidism in dogs.
\end{abstract}

Key words: Cryptorchidism, dog, laparoscopy (Source: CAB). 


\section{INTRODUCCIÓN}

El criptorquidismo es un defecto congénito común del tracto reproductivo de los perros $(1,2)$, y se reconoce como una condición heredada. A pesar de no existir suficientes datos sobre la ocurrencia de la alteración en el país, en la práctica clínica es usual encontrarla. También se conoce que los testículos retenidos tienen mayor riesgo de desarrollar neoplasias y presentar torsión (especialmente en los testículos neoplásicos) $(3,4)$. Debido a las implicaciones clínicas y la heredabilidad de la condición, se recomienda orquiectomía.

Es habitual que los pacientes con los testículos en el abdomen, se sometan a orquiectomía mediante laparotomía media ventral o paraprepucial $(1,5)$. Las técnicas quirúrgicas de mínima invasión son una alternativa adecuada para la realización de este procedimiento, debido a la reducción del trauma tisular y un menor dolor postoperatorio, además de la disminución en las complicaciones entre otras ventajas.

En Colombia y en general en todo el mundo, el desarrollo de las técnicas de mínima invasión todavía se encuentra muy limitado en cirugía de pequeños animales; sin embargo, el número de publicaciones al respecto se han multiplicado en los últimos años. Actualmente la disponibilidad de equipos en universidades y el interés de algunos veterinarios en desarrollar estos métodos, han permitido que el país incursione en el campo de la cirugía endoscópica en pequeños y grandes animales. A continuación se presentan dos casos de criptorquidectomía abdominal realizada mediante el uso de laparoscopia; se describen las técnicas empleadas, el manejo postoperatorio y las complicaciones manifestadas.

\section{Casos clínicos}

Dos perros criptórquidos se remitieron al servicio quirúrgico del Centro de Veterinaria y Zootecnia de la Universidad CES (Envigado, Colombia), para realizar orquiectomía. El primero de los pacientes, se trató de un Schnauzer Miniatura de 10 meses de edad y $4.7 \mathrm{~kg}$ de peso y el segundo paciente era un cruce poco común de Labrador y Pug, de 5 años de edad y un peso de $30 \mathrm{~kg}$.

En ninguno de los pacientes se detectaron anormalidades durante el examen físico excepto por la ausencia del testículo izquierdo en el escroto del paciente Schnauzer y de ambos testículos en el paciente cruzado. Tampoco se palparon las glándulas en el canal inguinal. En ambos casos se confirmó la presencia en el abdomen de los testículos no descendidos mediante ultrasonido (Figura 1).

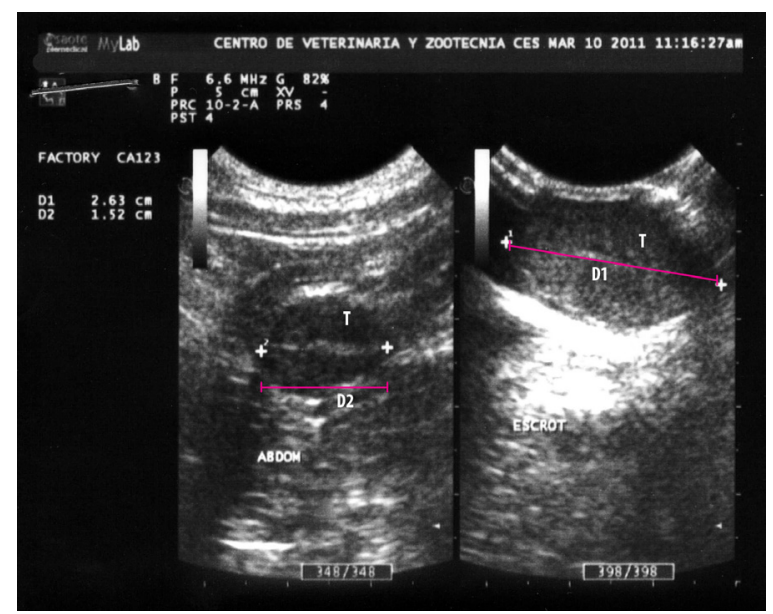

Figura 1. Ecografía abdominal. En el lado izquierdo de la foto se aprecia la imagen ecográfica del testículo retenido $(T)$ en la cavidad abdominal del paciente 1 (ABDOM). La imagen compara el eje largo del testículo retenido $(\mathrm{D} 2=1.52 \mathrm{~cm})$ vs. el testículo normal ( $T$ ) en el escroto (ESCROT) al lado derecho de la foto $(\mathrm{D} 1=2.63 \mathrm{~cm})$. La ecogenicidad del testículo retenido se consideró normal.

Los análisis hematológicos y bioquímicos de ambos pacientes estaban dentro de los parámetros normales (hemoleucograma, proteínas totales, creatinina, urea, alanina aminotransferasa [ALT] y fosfatasa alcalina [FA]).

Procedimientos quirúrgicos. Ambos perros tuvieron ayuno de 12 horas, luego de los cuales se premedicaron con acepromacina $(0.01 \mathrm{mg} / \mathrm{kg}$ intramusculares [IM]), tramadol (2 $\mathrm{mg} / \mathrm{kg}$ [IM]), dipirona (28 mg/kg intravenoso [IV]) y cefalotina (20 mg/kg [IM]. La medicación descrita se aplicó media hora antes del inicio del procedimiento quirúrgico y de acuerdo a la predilección del médico veterinario anestesiólogo.

La anestesia implicó la inducción mediante el uso de propofol ( $4 \mathrm{mg} / \mathrm{kg}$ [IV]) y se mantuvo con isofluorano en oxígeno después de la intubación traqueal. Ambos pacientes se depilaron desde el xifoides al pubis, se posicionaron en decúbito dorsal y se prepararon asépticamente.

Se realizó una técnica de laparoscopia total, mediante la inserción de tres puertos, previamente descrita por otros autores $(5,6)$. En principio, se creó el neumoperitoneo mediante la insuflación con $\mathrm{CO}_{2}$, utilizando la aguja de Veress insertada subumbilical luego de realizar una incisión de $2 \mathrm{~mm}$ en la piel sobre la línea media (Figura 2). La presión mantenida en la cavidad abdominal fue de $10 \mathrm{mmHg}$ en el Schnauzer y $12 \mathrm{mmHg}$ en el perro cruzado. 


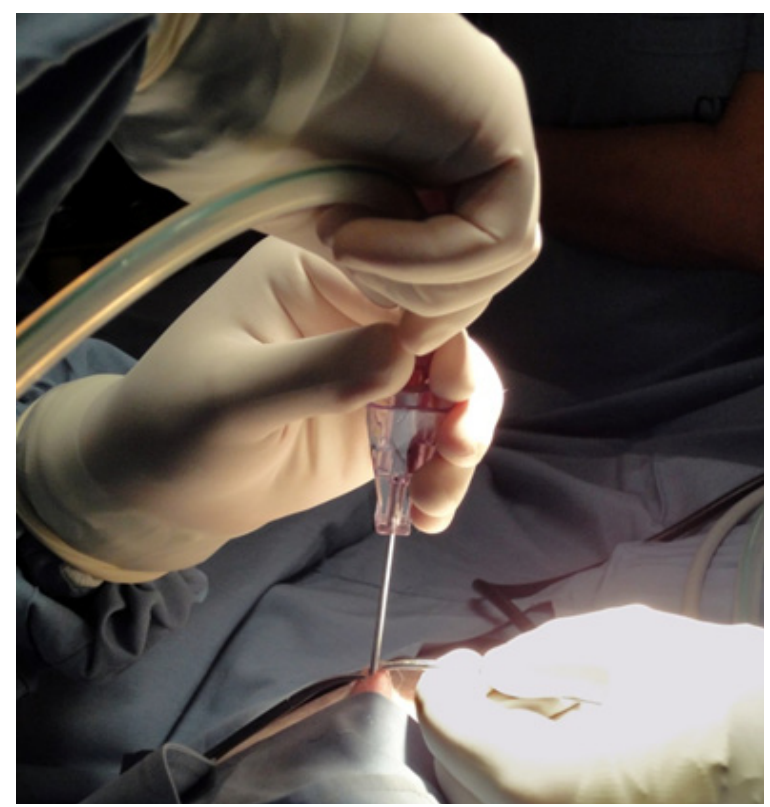

Figura 2. Creación del neumoperitoneo. Inserción de la aguja de Veress subumbilical para insuflar el abdomen con $\mathrm{CO}_{2}$ y permitir insertar de forma segura el primer trocar.

El primer puerto se insertó subumbilical en el mismo sitio de la punción con la aguja de Veress, y a través del trocar se introdujo un lente de 5 $\mathrm{mm}$ de diámetro, y de $10 \mathrm{~mm}$ en el segundo paciente; ambos lentes con ángulos oblicuos de $30^{\circ}$. Se establecieron otros dos puertos en posición paramedial lateral y caudal al primero, siguiendo los principios de triangulación. Una vez que los tres sitios fueron establecidos, ambos animales se posicionaron en Trendelenburg para permitir el movimiento del intestino hacia craneal y facilitar la visualización de los testículos (Figura 3).

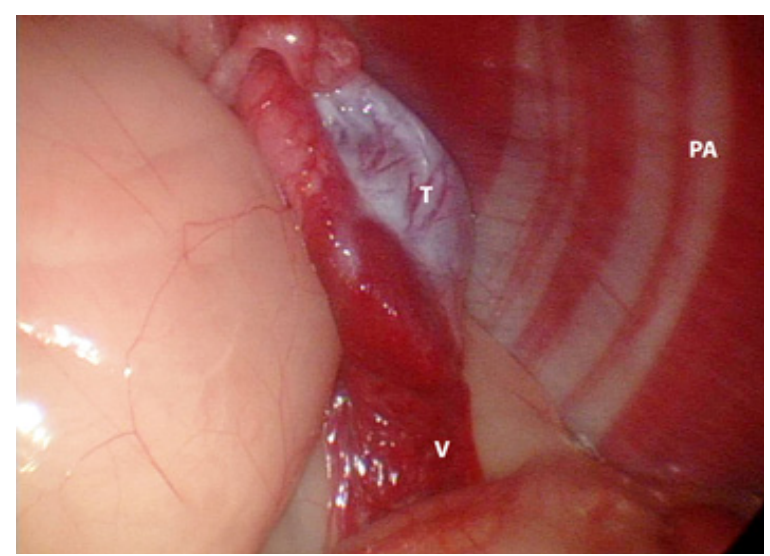

Figura 3. Identificación testicular en abdomen. Visualización del testículo derecho $(T)$ retenido en el paciente cruce Pug y Labrador una vez se posicionó en Trendelenburg. El testículo se encuentra próximo al anillo inguinal. Se aprecia la pared abdominal (PA) y la red de venas y arterias del testículo (V).
Cada testículo retenido se sujetó con pinzas laparoscópicas, y se retrajo hacia posición cráneo-ventral para facilitar su exposición (Figura 4). La pieza de mano del bisturí armónico conectada al generador (UltraCision Harmonic Scalpel Generator 300, Ethicon Endo-surgery, Cincinati, OH, USA) se insertó a través del puerto paramedial derecho y se utilizó para coagular y cortar el gobernáculo, cordón espermático y el pedículo vascular. En el segundo paciente, la posición de la pinza de prensión y el bisturí ultrasónico se revirtieron para repetir el procedimiento en el testículo contrario.

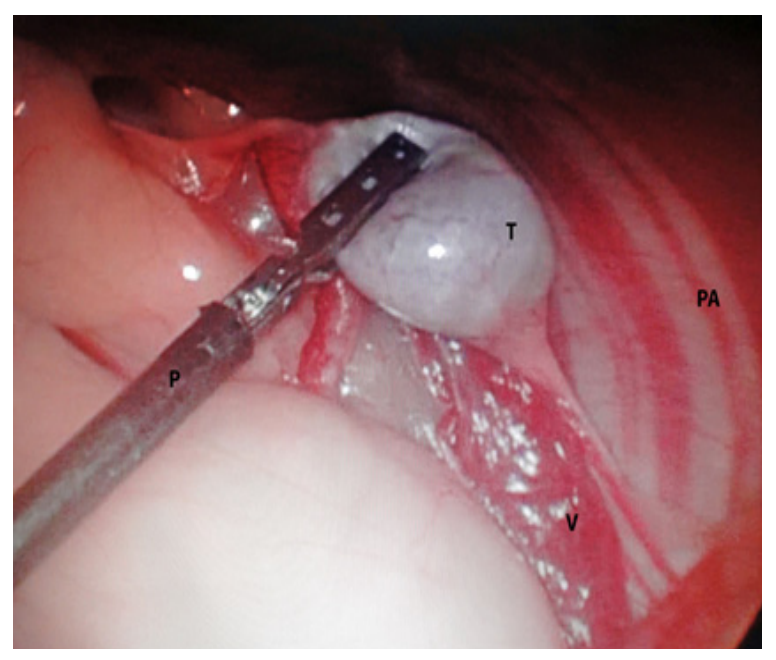

Figura 4. Retracción testicular. Elevación cráneo ventral del testículo ( $T$ ) con pinzas de retracción $(P)$ para facilitar la exposición y el corte posterior con el bisturí armónico. Se aprecia la pared abdominal (PA) y la red vascular (V).

Todos los sitios de corte se inspeccionaron para descartar hemorragia y seguidamente se introdujeron pinzas de Babcock y se sujetaron los testículos, permitiendo extraerlos a través de los orificios creados por las cánulas en el lado izquierdo. En el segundo paciente, se agrandó el sitio de incisión para permitir el paso de ambos testículos.

Una vez retirados los trocares y los testículos del abdomen, se procedió a descomprimir la cavidad abdominal aplicando presión sobre la pared y permitiendo la salida del gas a través de las incisiones. La fascia se suturó utilizando patrones simples separados de poliglactina 910 , de calibres 3-0 y 2-0 en el primero y segundo paciente respectivamente. Igual patrón y material se utilizó para el tejido subcutáneo, y las incisiones de la piel se suturaron con patrones simples separados de polipropileno 3-0. 
El testículo posicionado normalmente del paciente Schnauzer, se extrajo mediante orquiectomía preescrotal con técnica cerrada, empleando suturas absorbibles de poliglactina 910 calibre 3-0 para ligar el cordón espermático y las túnicas. El tejido subcutáneo se cerró con un patrón continuo de igual material y la piel se afrontó con un patrón subcuticular de polipropileno. No se presentó ninguna complicación intraquirúrgica.

Ambos pacientes se dieron de alta seis horas después de la recuperación anestésica, sin la necesidad del uso adicional de antibióticos. Se prescribieron analgésicos durante 3 días (meloxicam $0.1 \mathrm{mg} / \mathrm{kg} / 24 \mathrm{~h}$ ). No se reportaron complicaciones durante el posoperatorio inmediato. Una semana después de la cirugía, el paciente cruzado desarrolló un seroma en uno de los sitios de incisión (incisión izquierda), el cual al drenarse y ser limpiado, resolvió sin ninguna otra complicación. Las suturas no absorbibles de piel se retiraron diez días después del procedimiento.

\section{DISCUSIÓN}

El criptorquidismo es uno de los defectos congénitos más comunes que se aprecian en la práctica clínica de pequeñas especies animales y es la alteración congénita más común de los testículos en los perros $(1,2)$. Se trata de una condición que aparece con mayor frecuencia de forma unilateral y es el testículo derecho el más afectado (7).

La orquiectomía bilateral es el tratamiento de elección en estos pacientes, ya que el criptorquidismo se reconoce como una condición heredada, ligada al sexo autosómica recesiva de rasgo poligénico $(1,8)$. También los testículos retenidos se constituyen en factor de riesgo para el desarrollo de neoplasia testicular (13.6 a 14.3 mayor probabilidad de desarrollar tumores que los testículos escrotales) $(3,4)$, además, los testículos retenidos en el abdomen, presentan un riesgo incrementado de torsión cuando desarrollan neoplasias (9).

Existen varias técnicas para realizar orquiectomía en pacientes con los testículos retenidos en el abdomen, entre las cuales se encuentra la laparotomía media ventral o paraprepucial en el tercio caudal de la línea alba (10). Esta última es una técnica sencilla, pero puede representar un reto en caso de no identificar de forma inmediata la localización del testículo, lo que incrementa la manipulación del tejido y es una cirugía que con frecuencia causa dolor e inflamación de la zona paraprepucial. Otra técnica descrita implica el uso de pequeñas incisiones de laparotomía y la retracción mediante ganchos (esta técnica ya no se recomienda debido a las complicaciones descritas en algunos reportes) $(2,11)$.

También existe la posibilidad de utilizar técnicas de mínima invasión incluidas la criptorquidectomía abdominal laparoscópica total realizada en estos dos casos o la técnica asistida por laparoscopia que se ha descrito en publicaciones previas $(2,5,10)$.

La laparoscopia es una técnica de mínima invasión utilizada para observar las estructuras en la cavidad abdominal y que además permite la realización de múltiples procedimientos como la criptorquidectomía. La técnica involucra la distensión de la cavidad abdominal con gas (neumoperitoneo) y la utilización de laparoscopios rígidos colocados a través de puertos posicionados en la cavidad abdominal que permiten la introducción de instrumentos y lentes (12).

El acceso a la cavidad abdominal para la creación del neumoperitoneo en ambos casos se realizó a través de la inserción ciega al abdomen de la aguja de Veress (técnica cerrada), y posterior insuflación de $\mathrm{CO}_{2}$. Una vez insuflado el abdomen se insertó el trocar de forma segura en el abdomen dado el espacio creado con el $\mathrm{CO}_{2}$.

Para la inserción de la aguja de Veress, durante la criptorquidectomía, el cirujano realizó una incisión subumbilical con bisturí en la piel de unos $2 \mathrm{~mm}$ de longitud, el cual se disecó ligeramente para tunelizar a través del tejido subcutáneo. La aguja se posicionó en el sitio y se traccionó la piel alrededor para insertar la aguja. En ocasiones no se logra retraer la pared muscular de forma adecuada debido a la laxitud de la piel de los perros. Otra alternativa que utilizamos es la tracción de la pared mediante pinzas de campo a ambos lados del sitio de incisión, halando la pared muscular.

La aguja se inserta y se percibe el accionar del mecanismo de la aguja una vez se encuentra libre en la cavidad abdominal. Antes de iniciar el flujo de $\mathrm{CO}_{2}$, se asegura que la aguja se mueve suave y libremente. También se irriga una pequeña cantidad de solución salina fisiológica o Hartmann a través de la aguja, esta debe pasar sin dificultad o se deja caer unas gotas sobre la aguja y la presión intraabdominal negativa permite el flujo fácil de las gotas hacia la cavidad. También resulta útil elevar la pared abdominal y la lectura de presión abdominal (con el insuflador ya conectado) debe disminuir.

No se presentó ninguna complicación con la 
utilización de la técnica cerrada con aguja de Veress para la creación del neumoperitoneo. El autor también utiliza con frecuencia el acceso a la cavidad mediante minilaparotomía con posterior inserción del trocar e infusión del $\mathrm{CO}_{2}$ (técnica abierta o técnica de Hasson). Ambas técnicas se encuentran descritas (13) y son seguras si se realizan con la precaución debida. Las complicaciones asociadas a la inserción de los trócares incluye la perforación o laceración de órganos como el bazo, intestino delgado y grueso, la vejiga y grandes vasos (10).

Se tiene la creencia de que la introducción de la aguja de Veress puede causar mayores complicaciones por la posibilidad de ocasionar trauma con la aguja o al insertar el trocar. Estudios que comparan ambos accesos a la cavidad abdominal, han determinado que la entrada por técnica abierta está asociada con una reducción significativa en el número de fallas al ingresar, comparada con el acceso cerrado con aguja de Veress, sin embargo, no hay diferencia en la incidencia de daño vascular o visceral (14).

Otros métodos para acceder a la cavidad abdominal consisten en la introducción de trócares ópticos con o sin previa introducción del gas. También existe la posibilidad de la introducción ciega del primer trocar sin neumoperitoneo (13).

A través del primer puerto creado, se introdujo el instrumento óptico (lente) que permitió la inserción de los otros dos trócares con mínimo riesgo gracias a la asistencia visual. Durante el aprendizaje de la técnica de laparoscopia, es frecuente que los trócares se deslicen a través del espacio subcutáneo y creen espacios muertos que permitan la formación de seromas y enfisema subcutáneo, facilitado además por la laxitud de la piel del perro.

Una vez en la cavidad abdominal resulta evidente que la visualización del los testículos retenidos es muy fácil (comparada con la técnica abierta) y ayudada por la posición Trendelenburg a la que se sometieron ambos pacientes (Figura 3 ).

Tal como se ha comprobado en hembras caninas al realizar ovariohisterectomía mínimamente invasiva, es casi seguro que en el abordaje al abdomen en machos por laparoscopia para extraer los testículos retenidos, se reduzca el trauma de los tejidos, el dolor posoperatorio y las complicaciones durante la cicatrización, cuando se compara con las técnicas abiertas $(5,15-17)$. De hecho, resultó obvio para los dos casos presentados, que la técnica laparoscópica contribuyó con la reducción del tamaño de la incisión quirúrgica que suele ser paraprepucial cuando se realiza por laparotomía y que puede ser una herida dolorosa y molesta para los pacientes. También la técnica permitió en estos dos casos, una cirugía y recuperación muy rápidas, dada la facilidad para manipular los testículos retenidos mediante las pinzas laparoscópicas. Los perros no exhibieron signos clínicos significativos de dolor durante el postoperatorio. De cualquier modo, aunque sea probable que haya reducción del dolor comparado con la cirugía abierta, es importante que se realicen estudios que lo comprueben para la criptorquidectomía abdominal.

En seres humanos y animales, la laparoscopia causa malestar en los pacientes $(16,18)$. El origen del dolor post-laparoscopia en humanos se puede clasificar en tres aspectos: visceral, incisión y dolor en el hombro (este último asociado posiblemente a la sobredistensión de las fibras musculares del diafragma) (18). Los trabajos realizados en caninos, demuestran la reducción en el dolor comparado con las técnicas abiertas; sin embargo no se conocen trabajos que identifiquen dolor visceral o de otro tipo asociado al pneumoperitoneo.

No se presentaron complicaciones aparentes asociadas al uso del $\mathrm{CO}_{2}$ para el mantenimiento del neumoperitoneo en los casos presentados. El $\mathrm{CO}_{2}$ no es combustible, permitiendo el uso de electrocauterización y además, su alta solubilidad permite la expiración sencilla a través de los pulmones. Otros gases que se han empleado incluyen aire, óxido nitroso, helio, argón y oxígeno (19).

Otros procedimientos de laparoscopia total o asistidos se encuentran descritos en pequeños animales, entre los que se encuentran cirugía reproductiva en machos y hembras (ovariectomía, ovariohisterectomía, criptorquidectomía) $(5,15,20)$. También otras técnicas como gastropexia, cistotomía, y biopsias de órganos (21-23), y recientemente métodos más avanzados como adrenalectomía, colecistectomía y lobectomía pulmonar por toracoscopia (24-26). Con respecto a la criptorquidectomía laparoscópica, se han descrito varios procedimientos usando 3 puertos $(5,10)$ tal y como se realizó en este caso, o mediante dos puertos (27), y también se han reportado técnicas asistidas $(2,5)$.

Al igual que en cirugía abierta, las técnicas mínimamente invasivas requieren el uso de métodos para control del sangrado. El uso del bisturí ultrasónico como técnica de hemostasia en los casos mencionados, no requirió la utilización de otros métodos adicionales en ninguna de 
las dos cirugías, contribuyendo con la rapidez del procedimiento y una manipulación mínima de los tejidos. Entre las ventajas evidentes del uso del bisturí armónico se encuentra que no se requiere de un entrenamiento avanzado ya que se maneja de forma similar al electrobisturí y que puede obviar el uso de ligaduras que retrasan la duración del procedimiento. De hecho, en otros procedimientos de criptorquidectomía abdominal laparoscópica realizados por el autor con la utilización de nudos extracorpóreos, fue notorio el incremento del tiempo quirúrgico. Es definitivo que el método más accesible en costos es el uso de nudos intra o extracorpóreos; sin embargo, requieren mayor entrenamiento y retrasan el tiempo quirúrgico cuando se compara con el uso del dispositivo ultrasónico.

El bisturí ultrasónico empleado es un instrumento quirúrgico que, mediante una pinza, corta y coagula simultáneamente (28). En la interface entre la pinza y el tejido, se incrementa la temperatura y ocurren cavitaciones, y la fricción ultrasónica induce la desnaturalización de las proteínas provocando el corte de los tejidos al mismo tiempo que causa un sellado de los bordes y un cierre vascular de estructuras iguales o menores a $5 \mathrm{~mm}$ de diámetro (16).

Otra ventaja del bisturí armónico es que transfiere menos energía a los tejidos que cuando se utilizan dispositivos de alta frecuencia o láser, y por lo tanto hay menos posibilidad de daño térmico o penetración profunda ya que es capaz de mantener el proceso a una temperatura de alrededor de $60^{\circ} \mathrm{C}$ y no se presentan quemaduras sobre la superficie y los tejidos circundantes $(28,29)$. La desventaja más importante de los dispositivos es su alto costo (30), más aún considerando los bajos precios que se suelen cobrar por los servicios veterinarios en algunos países latinoamericanos. La utilización del dispositivo ultrasónico ya se ha reportado en medicina veterinaria de pequeños animales, especialmente en la realización de técnicas de ovariohisterectomía $(16,31)$.

Otras técnicas de hemostasia están descritas en laparoscopia en seres humanos y animales, entre los cuales se destaca el uso de anudamientos (extracorpóreos e intracorpóreos), aplicación de clips metálicos y diversos instrumentos electroquirúrgicos (incluidos los electrobisturí mono y bipolar), coaguladores a gas argón, bisturíes por radiofrecuencia y por ultrasonido como el empleado en este caso $(20,28)$.

En el caso del paciente cruzado, el perro desarrolló un seroma como complicación posquirúrgica. En medicina veterinaria son pocos los casos o series de casos publicados relacionados con las complicaciones durante o posterior a cirugía por laparoscopia. Durante el procedimiento, éstas pueden estar asociadas a la anestesia (hipotensión y compromiso cardiovascular), al mantenimiento del neumoperitoneo, desajustes en los equipos, hemorragia excesiva, perforaciones de vísceras y complicaciones respiratorias por émbolos (32). Las complicaciones intraquirúrgicas reportadas varían del $2 \%$ hasta el $35 \%$ y la mayoría consisten en sangrados menores. Las complicaciones posquirúrgicas pueden incluir hemorragia y anemia, peritonitis, hipotensión, metástasis u otras complicaciones en los sitios de colocación de puertos, tales como enfisema subcutáneo, dehiscencias, hernias e infección de la herida quirúrgica. Los seromas como el presentado en el segundo caso, también se han reportado y representan desde el 3.1\% hasta el $5 \%$ en dos estudios presentados $(31,32)$ y suelen resolverse con un manejo mínimo. Una vez realizado el drenaje y limpieza, el paciente resolvió sin presentar ninguna complicación adicional.

El desarrollo de seromas puede estar asociado a la disección accidental del tejido que sucede al tratar de insertar el trocar en la cavidad abdominal y se desliza a través del espacio subcutáneo, evento que sucede ocasionalmente especialmente en las primeras fases del entrenamiento en laparoscopia. En este caso no hubo dificultades en la inserción del trocar, sin embargo, el sitio donde se presentó el seroma estaba relacionado con el puerto por el cual se extrajeron los dos testículos retenidos del paciente cruce. Es posible que la manipulación del sitio al extraer las glándulas haya contribuido con la presentación del seroma.

Aunque no se han comparado las diferencias entre criptorquidectomía laparoscópica con la técnica abierta en estudios de investigación, en humanos, varios procedimientos de laparoscopia presentan menos complicaciones al comparar con la técnica abierta, además, de reducir el uso de analgésicos, la hospitalización y favorecer un menor tiempo de licencia posoperatorio.

En la actualidad, las técnicas laparoscópicas no se utilizan de manera extensiva por los veterinarios a nivel global $(33,34)$; sin embargo, su uso ha aumentado y las publicaciones se han acrecentado en años recientes (35). En Colombia, la técnica de cirugía mínimamente invasiva en medicina veterinaria de pequeños animales, no se encuentra desarrollada y el autor sólo conoce una publicación local describiendo una técnica de ovariohisterectomía laparoscópica (31). En equinos también se están realizando procedimientos laparoscópicos en el país, 
tales como criptorquidismos abdominales y correcciones de hernias inguinales (casos no publicados).

Entre las razones para el limitado uso de las técnicas mínimamente invasivas se encuentra el costo y complejidad de los equipos, se requiere entrenamiento especializado y es necesaria la asistencia quirúrgica por otra persona entrenada en instrumentación quirúrgica. También es importante tener unas instalaciones apropiadas, ya que se requiere una logística y un amplio espacio para acomodar y manejar el equipo completo (30). Es posible que exista una falta de interés de los propietarios en invertir un costo mayor de cirugía al comparar con las técnicas tradicionales, pero la técnica comienza a difundirse. Otros procedimientos realizados por laparoscopia incluyen exploración abdominal, de tórax, y biopsias de órganos entre otros.

En conclusión, las técnicas de cirugía de mínima invasión se constituyen en una alternativa ideal para la realización de procedimientos quirúrgicos en pequeños animales y posiblemente se convertirán en el estándar de realización de una gran variedad de procedimientos quirúrgicos en animales, por el hecho de reducir el trauma a los tejidos, el dolor y algunas de las complicaciones que se presentan en cirugía convencional.

En la realización de criptorquidectomía abdominal en perros, la cirugía de mínima invasión es una excelente alternativa que reduce el dolor postoperatorio y la manipulación de los tejidos y se realiza de forma rápida y segura.

\section{REFERENCIAS}

1. Birchard SJ, Nappier M. Cryptorchidism. Compend Contin Educ Vet 2008; 30(6):325-336.

2. Miller NA, Van Lue SJ, Rawlings CA. Use of laparoscopic-assisted cryptorchidectomy in dogs and cats. J Am Vet Med Assoc 2004; 224(6):875-8.

3. Liao AT, Chu P-Y, Yeh L-S, Lin C-T, Liu C-H. A 12year retrospective study of canine testicular tumors. J Vet Med Sci 2009; 71(7):919-23.

4. Ortega-Pacheco A, Rodríguez-Buenfil JC, Segura-Correa JC, Bolio-Gonzalez ME, Jiménez-Coello $M$, Linde Forsberg $C$. Pathological conditions of the reproductive organs of male stray dogs in the tropics: prevalence, risk factors, morphological findings and testosterone concentrations. Reprod Domest Anim 2006; 41(5):429-37.

5. Mayhew P. Surgical views: laparoscopic and laparoscopic-assisted cryptorchidectomy in dogs and cats. Compend Contin Educ Vet 2009; 31(6):274-81.

6. Lew M, Jałyński M, Kasprowicz A, Brzeski W. Laparoscopic cryptorchidectomy in dogs --report of 15 cases. Pol J Vet Sci 2005; 8(3):251-4.

7. Yates D, Hayes G, Heffernan M, Beynon R. Incidence of cryptorchidism in dogs and cats. Vet Rec 2003; 152(16):502-4.
8. Cox VS, Wallace LJ, Jessen CR. An anatomic and genetic study of canine cryptorchidism. Teratology 1978; 18(2):233-40.

9. Pearson $\mathrm{H}$, Kelly DF. Testicular torsion in the dog: a review of 13 cases. Vet Rec 1975; 97(11):200-4.

10. Urbanová L, Crha M, RaušerP, Lorenzová J, Nečas A. Laparoscopically Assisted Cryptorchidectomy Using LigaSure $\mathbb{R}$ Electrocoagulation. Acta Vet Brno 2010; 79(2):313-8.

11. Schulz KS, Waldron DR, Smith MM, Henderson RA, Howe LM. Inadvertent prostatectomy as a complication of cryptorchidectomy in four dogs. J Am Anim Hosp Assoc 1996; 32(3):211-4.

12. Monnet E, Twedt DC. Laparoscopy. Vet Clin North Am Small Anim Pract 2003; 33(5):1147-63.

13. Sartori CA, Balduino M. Creación del neumoperitoneo e inserción de los trócar. Cirugía miniinvasiva del tórax y abdomen. Edición año 2010. Milano - Italia: Elsevier Masson S:R:L; 2010.

14. Ahmad G, Duffy JMN, Phillips K, Watson A. Laparoscopic entry techniques. Cochrane Database Syst Rev 2008; (2):CD006583.

15. Davidson EB, David moll $H$, Payton ME. Comparison of Laparoscopic Ovariohysterectomy and Ovariohysterectomy in Dogs. Vet Surg 2004; 33(1):62-9. 
16. Hancock RB, Lanz OI, Waldron DR, Duncan RB, Broadstone RV, Hendrix PK. Comparison of Postoperative Pain After Ovariohysterectomy by Harmonic Scalpel-Assisted Laparoscopy Compared with Median Celiotomy and Ligation in Dogs. Vet Surg 2005; 34(3):273-82.

17. Culp WTN, Mayhew PD, Brown DC. The Effect of Laparoscopic Versus Open Ovariectomy on Postsurgical Activity in Small Dogs. Vet Surg 2009; 38(7):811-7.

18. Sandhu T, Yamada S, Ariyakachon V, Chakrabandhu T, Chongruksut W, Ko-iam W. Low-pressure pneumoperitoneum versus standard pneumoperitoneum in laparoscopic cholecystectomy, a prospective randomized clinical trial. Surg Endosc 2009; 23(5):1044-7.

19. Pappas TN, Fecher AM. Principles of minimally invasive surgery. Surgery : basic science and clinical evidence. New York, NY: Springer; 2008.

20. Mayhew PD, Brown DC. Comparison of three techniques for ovarian pedicle hemostasis during laparoscopic-assisted ovariohysterectomy. Vet Surg 2007; 36(6):541-7.

21. Rothuizen J, Twedt DC. Liver biopsy techniques. Vet Clin North Am Small Anim Pract 2009; 39(3):469-80.

22. Rawlings CA. Laparoscopic-assisted gastropexy. J Am Anim Hosp Assoc 2002; 38(1):15-9.

23. Rawlings CA, Mahaffey MB, Barsanti JA, Canalis C. Use of laparoscopic-assisted cystoscopy for removal of urinary calculi in dogs. J Am Vet Med Assoc 2003; 222(6):759-61, 737.

24. Jiménez Peláez $M$, Bouvy BM, Dupré GP. Laparoscopic adrenalectomy for treatment of unilateral adrenocortical carcinomas: technique, complications, and results in seven dogs. Vet Surg 2008; 37(5):444-53.

25. Mayhew PD, Mehler SJ, Radhakrishnan A. Laparoscopic cholecystectomy for management of uncomplicated gall bladder mucocele in six dogs. Vet Surg 2008; 37(7):625-30.
26. Lansdowne JL, Monnet E, Twedt DC, Dernell WS. Thoracoscopic lung lobectomy for treatment of lung tumors in dogs. Vet Surg 2005; 34(5):530-5.

27. Martínez JM, Granados JR, Mateo MB. Uso de la laparoscopia en criptórquido abdominal unilateral. Técnica con dos puertos. Centro Veterinario 2010; (42):10-4.

28. Iongoni M, Bottero L, Faillace G, Rota E. Instrumental laparoscópico. Cirugía miniinvasiva del tórax y abdomen. Edición año 2010. Milano - Italia: Elsevier Masson S:R:L; 2010.

29. Perko Z, Pogorelić Z, Bilan K, Tomić S, Vilović $K$, Krnić D, et al. Lateral thermal damage to rat abdominal wall after harmonic scalpel application. Surg Endosc 2006; 20(2):322-4.

30. Van Lue SJ, Van Lue AP. Equipment and Instrumentation in Veterinary Endoscopy. Veterinary Clinics of North America: Small Animal Practice 2009; 39:817-37.

31. Ruiz I, Acevedo C, Rodriguez M. Descripción y evaluación de una técnica de ovariohisterectomía laparoscópica en perras sanas. Rev Colom Cienc Pecu 2008; 21(4):546-58.

32. McClaran JK, Buote NJ. Complications and need for conversion to laparotomy in small animals. Vet Clin North Am Small Anim Pract 2009; 39(5):941-51.

33. Devitt CM, Cox RE, Hailey JJ. Duration, complications, stress, and pain of open ovariohysterectomy versus a simple method of laparoscopic-assisted ovariohysterectomy in dogs. J Am Vet Med Assoc 2005; 227(6):921-7.

34. Mayhew P. Developing minimally invasive surgery in companion animals. Vet Rec de 2011; 169(7):177-8.

35. Matyjasik H, Adamiak Z, Pesta W, Zhalniarovich Y. Laparoscopic procedures in dogs and cats. Pol J Vet Sci 2011; 14(2):305-16. 